\title{
Chorea Induced by Low-Dose Trazodone
}

\author{
Alisdair McNeill \\ Central Newcastle Medical SHO Rotation, Freeman Hospital and Royal Victoria Infirmary, \\ Newcastle-upon-Tyne, UK
}

Dear Sir,

Trazodone is an atypical anti-depressant with prominent sedative properties. It has a complex pharmacology, with trazodone being an antagonist at $5-\mathrm{HT}_{2}$ receptors, while its active metabolite $m$-chlorophenylpiperazine ( $m$-CPP) is a 5-HT agonist [1]. During treatment plasma levels of $m$-CPP can exceed those of trazodone, and it is unclear whether 5-HT agonist or antagonist effects dominate in vivo [1]. Here the first case of chorea associated with trazodone use is reported.

\section{Case Report}

A 78-year-old female was admitted with generalised, symmetrical choreiform movements of all 4 limbs accompanied by involuntary grimacing and tongue protrusion. Three days prior to admission she had been commenced on trazodone $50 \mathrm{mg}$ nocte and chlormethiazole $384 \mathrm{mg}$ nocte for insomnia. The abnormal movements had begun on the day of admission. Control of the chorea was achieved with diazepam $(5 \mathrm{mg}$ TDS), and the abnormal movements resolved 6 days after withdrawal of trazodone and cessation of diazepam. Physical examination, including a comprehensive neurological evaluation, was unremarkable apart from an MMSE of $21 / 30$. There were no features of a depressive, psychotic or obsessive-compulsive disorder. The past medical history included a coronary artery bypass and mitral valve repair at the age of 73 and rheumatic fever with Sydenham's chorea at the age of 15 . There was no family history of neurological disorders and the patient was not taking other medications known to cause movement disorders. CT brain and chest radiograph were normal. It was decided not to perform MRI brain as it was felt that the probability of an ischaemic lesion not visualised by CT was very low. Routine bloods demonstrated no abnormalities in the full blood count, liver function or renal function. The patient had been noted to be hyponatraemic for at least 2 years (around 125-130 mmol/l), but her plasma osmolarity was normal and there had been no recent changes in her plasma sodium levels. ESR was $8 \mathrm{~mm} / \mathrm{h}$. Thyroid function, blood glucose, calcium, magnesium, phosphate, serum caeruloplasmin, vitamin D and parathyroid hormone levels were all normal. The anti-streptolysin $\mathrm{O}$ titre was normal $(<200 \mathrm{U} / \mathrm{ml})$. Anti-nuclear antibody (titre 1:160) and anti-cardiolipin antibodies were positive, but the patient did not meet the American College of Rheumatology criteria for SLE or anti-phospholipid antibody syndrome and was anti-dsDNA negative with normal serum complement. Anti-basal ganglia antibodies were negative. Given the late age of onset and lack of family history it was decided not to perform genetic testing for Huntington's disease. CA-125 was elevated but pelvic ultrasound and $\mathrm{CT}$ abdomen demonstrated no ovarian or other malignancy. The patient was discharged 12 days after admission with no further occurrence of chorea.

\section{Discussion}

Chorea is a hyperkinetic movement disorder characterised by irregular, flowing, non-stereotyped involuntary movements
[2]. It can be subdivided into primary (inherited, e.g. Huntington's chorea) or secondary cases (immune, infectious, drug induced, paraneoplastic). Chorea is proposed to result from abnormal phasic activity within the globus pallidus pars internus that results in involuntary activation of thalamo-cortical motor circuits and abnormal movements [2]. Pharmacologically chorea is associated with excessive dopaminergic neurotransmission [2].

Movement disorders associated with trazodone use are rarely reported. A literature review found just 2 cases of parkinsonism [3, 4], 2 cases of dystonia [5, 6] and a case of myoclonus [7] associated with trazodone. There are no published cases of trazodone-induced chorea. However, the relationship between initiation of trazodone therapy with onset of chorea and withdrawal of trazodone with remission of chorea in this case argues that the dyskinesias were trazodone induced. The mechanism of trazodone-induced chorea is unclear. There is evidence from murine studies that low-dose trazodone enhances dopaminergic neurotransmission by antagonising $5-\mathrm{HT}_{2 \mathrm{C}}$ receptors, which normally exert tonic inhibition of dopamine neurotransmission in the basal ganglia [8]. It is possible in the case described here that low-dose trazodone, acting on basal ganglia damaged by rheumatic fever, induced dysregulated dopaminergic neurotransmission and choreiform movements. However, as dyskinesias can be induced by several classes of drugs, acting on distinct neurotransmitter systems, and as trazodone influences multiple neurotrans-

\section{KARGER}

Fax +4161306 1234 E-Mail karger@karger.ch www.karger.com
C 2006 S. Karger AG, Basel 0014-3022/06/0552-0101\$23.50/0

Accessible online at: www.karger.com/ene
Dr. Alisdair McNeill

11 Broomieknowe Park

Bonnyrigg, EH19 2JB (UK)

E-Mail amcneill@doctors.org.uk 
mitters [1], the pharmacology of trazodoneinduced chorea is likely to feature more than simply dysregulation of dopamine transmission. The study of Luparini et al. [9] demonstrated that low concentrations of trazodone inhibit GABA release and delay release of 5-HT from cultured neurons. This may facilitate dyskinesias directly by reducing inhibitory neurotransmission through GABA and indirectly by attenuating 5-HT release. In contrast, higher doses of trazodone may inhibit dyskinesias, with the study of Balsara et al. [8] demonstrating high doses of trazodone inhibit dopaminergic neurotransmission. This hypothesis is supported by clinical research reporting that trazodone can be used to treat dyskinesias including chorea [10]. Future research might profitably investigate high-dose (150-300 mg) trazodone usage in the treatment of hyperkinetic movement disorders.

\section{References}

1 Anonymous: Mood disorders; in Gelder M, Mayour R, Cowen P (eds): Shorter Oxford Textbook of Psychiatry, ed 4. Oxford, Oxford University Press, 2001, pp 269-327.

2 Mink J: The basal ganglia and involuntary movements: impaired inhibition of competing motor patterns. Arch Neurol 2003;60:13651368.

3 Fukunishi I, Kitaoka T, Shirai T, et al: A hemodialysis patient with trazodone-induced parkinsonism. Nephron 2002;90:222-223.

4 Albanese A, Rossi P, Altavista MC: Can trazodone induce parkinsonism? J Clin Neuropharmacol 1988;11:180-182.

5 Lewis CF, DeQuardo JR, Tandon R: Dystonia associated with trazodone and setraline. J Clin Psychopharmacol 1997;17:64-65.

6 Kramer MS, Marcus DJ, DiFerdinando J, Dewey D: Atypical dystonia associated with trazodone treatment. J Clin Pharmacol 1986; 6:117-118.
7 Patel HC, Bruza D, Yeragani V: Myoclonus with trazodone. J Clin Psychopharmacol 1988; 8:152.

8 Balsara JJ, Jadhav SA, Gaonkar RK, et al: Effects of the antidepressant trazodone, a 5-HT $2 \mathrm{~A} / 2 \mathrm{C}$ receptor antagonist, on dopamine-dependent behaviour in rats. Psychopharmacology 2005; 179:597-605.

9 Luparini MR, Garrone B, Pazzagli M, et al: A cortical GABA-5HT interaction in the mechanism of action of the antidepressant trazodone. Prog Neuropsychopharmacol Biol Psychiatry 2005;28:1117-1127.

10 Rubino R, Provera P, Gilardengo P, et al: Trazodone in involuntary pathologic movements. Riv Neurol 1984;54:52-73. 